\title{
Directed Differentiation of Pluripotent Stem Cells: From Developmental Biology to Therapeutic Applications
}

\author{
S. Irion, M.C. Nostro, S.J. Kattman, ANd G.M. Keller \\ McEwen Centre for Regenerative Medicine, University Health Network, Toronto, Ontario M5G 1L7, Canada
}

\begin{abstract}
The discovery of human pluripotent stem cells has laid the foundation for an emerging new field of biomedical research that holds promise to develop models of human development and disease, establish new strategies for discovering and testing drugs, and provide systems for the generation of cells and tissues for transplantation for the treatment of disease. The remarkable potential of pluripotent stem cells has sparked interest and excitement in academia, the biotechnology and pharmaceutical industries, as well as the lay public. Although the potential of human pluripotent stem cells is truly outstanding, fulfilling this potential is solely dependent on our ability to efficiently generate functional cell types from them. Some of the most successful approaches in this area to date are those that have applied the principles of developmental biology to stem cell differentiation. In this chapter, we review these concepts and highlight specific examples demonstrating that pluripotent stem cell differentiation in culture recapitulates the key aspects of early embryonic development. By continuing to translate insights from embryology to stem cell biology, progress in our ability to generate specific cell types from pluripotent stem cells will advance, yielding enriched populations of human cell types, including cardiomyocytes, hematopoietic cells, hepatocytes, pancreatic $\beta$ cells, and neural cells, for drug discovery, functional evaluation in preclinical models of human disease, and ultimately clinical applications.
\end{abstract}

Although early insights into pluripotent stem cells (PSCs) were provided by studies using embryonal carcinoma cells isolated from teratocarcinomas (Stevens and Little 1954), realization of the true potential of such stem cells came with the discovery of mouse embryonic stem cells (mESCs) (Evans and Kaufman 1981; Martin 1981). The initial studies with mESCs rapidly established the defining characteristics of PSCs, namely, their ability to proliferate for extended periods of time in an undifferentiated state in culture and their capacity to generate derivatives of the three primary germ layers. Within a decade of the discovery of mESCs, technologies for their genetic alteration through homologous recombination (HR) were developed, enabling the routine generation of strains of mice carrying specific mutations (Glaser et al. 2005). The potential of mESCs was also tested in vitro in studies that demonstrated that these stem cells were able to generate a variety of mature cell types following differentiation in culture (Keller 2005). Although most early experiments used crude culture conditions that resulted in the generation of mixed lineage populations, they provided a clear demonstration that the in vitro differentiation system could be used as a model for studies on mammalian development and provided the basis for the widely held notion that PSCs may some day represent a novel source of cell types and tissues for future pharmaceutical and clinical applications (Fig. 1).

These theoretical applications moved a significant step closer to practice with the isolation of human embryonic stem cells (hESCs) (Thomson et al. 1998). Although hESCs appear to represent a slightly different stage of development and respond to different signaling pathways than their mouse counterparts, they do display the key characteristics of PSCs. hESCs can be maintained and expanded in an undifferentiated state in culture and are able to generate progeny of the three primary germ layers when induced to differentiate in culture or to form teratomas in vivo (Thomson et al. 1998). The fact that hESCs display pluripotency was critical because it fulfilled the requirement of a stem cell population that could potentially give rise to unlimited supplies of functional human cells appropriate for transplantation, drug discovery, and developmental biology. A major hurdle that remained with the use of hESCs as a source of "donor" tissue for transplantation was allogeneic graft rejection. Efforts to overcome this obstacle included establishing banks of hESCs and generating patient-specific PSCs through somatic cell nuclear transfer or genetic reprogramming of somatic cells.

The landmark studies of Takahashi et al. (Takahashi and Yamanaka 2006) demonstrated the feasibility of the latter approach and showed that transient expression of four transcription factors normally found in ESCs led to the reprogramming of mouse embryonic fibroblasts (MEFs) to a pluripotent state. These reprogrammed cells were known as induced PSCs (iPSCs). Mouse iPSCs (miPSCs) have molecular profiles similar to those of mESCs and display pluripotent potential and the capacity to proliferate and expand in an undifferentiated state in culture (Takahashi and Yamanaka 2006). Following the discovery of miPSCs, human iPSCs (hiPSCs) were generated from fibroblasts using a similar strategy of transient expression of pluripotency genes (Takahashi et al. 2007; Yu et al. 2007; Park et al. 2008). hiPSCs could be maintained in a undifferentiated state in culture and were able to generate progeny of the three primary germ layers, documenting their pluripotency. Since the initial discovery of miPSCs and hiPSCs, significant advances have been made in expanding the target cell types that can be reprogrammed as well as in developing nonviral approaches for delivery 


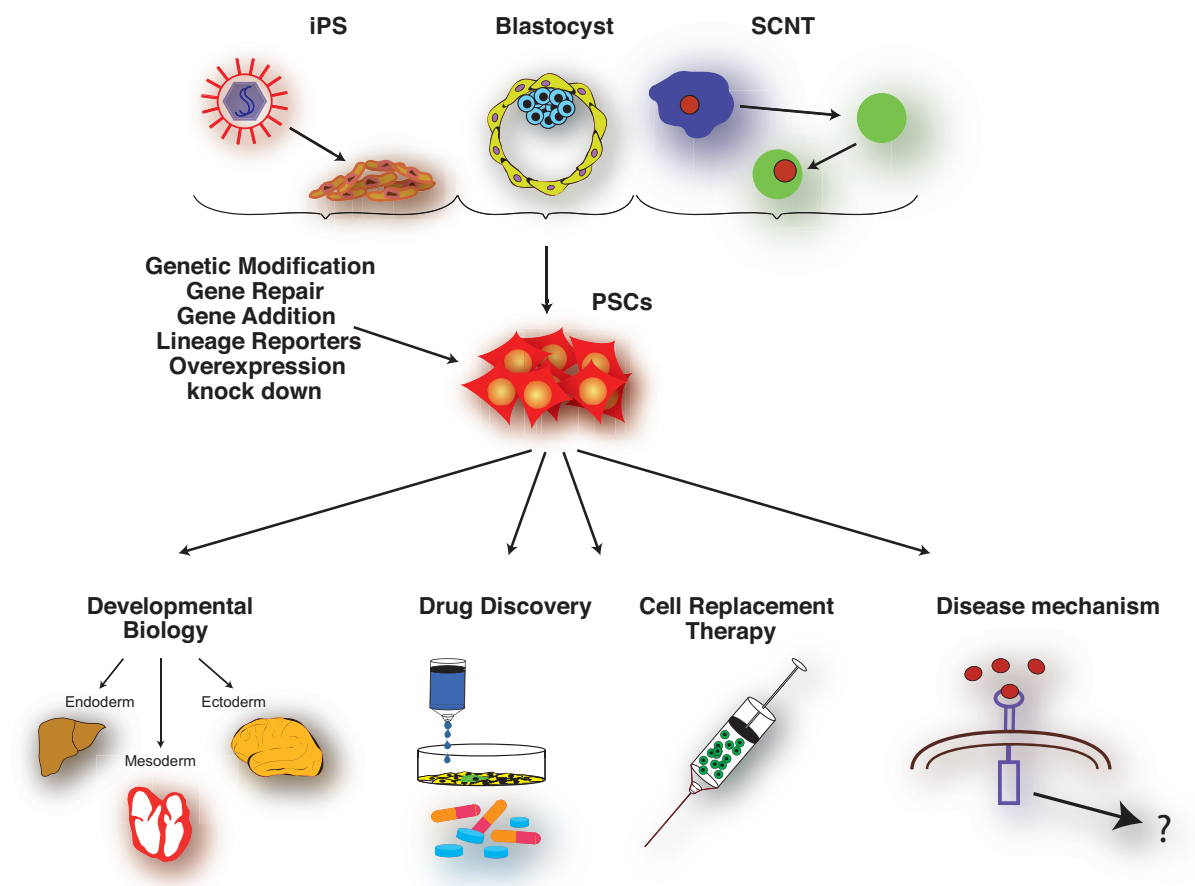

Figure 1. Pluripotent stem cell sources and their application. PSCs can be generated from the inner cell mass of the mammalian blastocyst from a normal fertilized egg, from an egg that has undergone somatic cell nuclear transfer, or by reprogramming somatic cells with pluripotency factors. Unmanipulated PSCs or those that have been genetically modified can be used for developmental biology studies, drug discovery and drug testing, cell replacement therapy, or modeling human diseases in culture.

of the reprogramming factors ( $\mathrm{Li}$ et al. 2009). Given the pace of progress in this field, it is likely that technologies for reprogramming somatic cells in a safe and controlled manner will be developed in the near future. Combined with the establishment of animal-free culture systems, the creation of clinically compliant patient-specific PSCs could soon be a reality (Fig. 1).

With the availability of hESCs, mESCs, and iPSCs (hereafter referred to as mouse and human pluripotent stem cells; mPSCs and hPSCs), the challenge facing the field is the development of strategies for the generation of homogeneous populations of functional cell types from them. Such strategies must be reliable, scalable, and applicable to a broad range of stem cells. As different cell types are generated, appropriate assays need to be developed to evaluate their function. For cell-based therapy, predictive preclinical models of human disease must be used and assays need to be in place to demonstrate that the transplanted population has integrated into the graft site and that the cells are functioning as expected. Drug discovery and screening applications will require a constant supply of relatively pure cell populations at comparable stages of development to provide reproducibility in the various assays. When establishing models of human disease from patient-specific iPSCs, the generation of the appropriate lineages possibly in the context of a threedimensional organ-like structure will be required. For all applications, the maturation stage of the target population will be an important factor in determining the success of the approach. The demands of these different applications highlight the need for studies aimed at understanding the mechanisms that control the induction, expansion, and maturation of specific cell types from PSCs.

In this chapter, we review the early events regulating mammalian development, including germ-layer specification, and illustrate how the principles of developmental biology have been successfully applied to strategies for the directed differentiation of PSCs. In the final section, we conclude with a discussion of applications for the genetic modification of PSCs.

\section{MOUSE GASTRULATION: FORMATION OF THE PRIMITIVE STREAK AND THE PRIMARY GERM LAYERS}

The primary germ layers, the founder populations of all cell types in the body, are established early in embryonic development, during a process known as gastrulation (Tam and Behringer 1997). At the onset of gastrulation, a transient structure known as the primitive streak (PS) forms in the region of the epiblast that will ultimately give rise to the posterior part of the embryo (Fig. 2). As gastrulation proceeds, epiblast cells migrate through the PS and exit as populations fated to either the mesoderm or definitive endoderm cell lineages. Gene expression and lineage-tracing studies have been used to define three main regions of the PS: the posterior, mid, and anterior regions. With respect to gene expression patterns, brachyury $(t)$ (Kispert and Herrmann 1994) and mixll (Hart et al. 2002) are found throughout the entire PS, 
foxa2 and goosecoid (Sasaki and Hogan 1993; Kinder et al. 2001) mark the anterior region, and hoxbl and evxl define the posterior region (Dush and Martin 1992; Forlani et al. 2003). The movement of uncommitted epiblast cells through the PS is controlled both spatially and temporally. The first cells to move through this structure do so in the posterior region and give rise to the extraembryonic mesoderm that forms the allantois and amnion as well as the hematopoietic, endothelial, and vascular smooth muscle cells of the yolk sac (Parameswaran and Tam 1995; Kinder et al. 1999). The next populations of epiblast cells traverse the PS in progressively more anterior sites, contributing to cranial, cardiac, paraxial, and axial mesoderm, respectively. Finally, cells that migrate through the most anterior part of the PS give rise to the definitive endoderm. Epiblast cells fated to the third germ layer, ectoderm, lie in the anterior region of the early embryo and do not migrate through the PS.

Although the mechanisms controlling PS formation and germ-layer induction are not fully understood, targeting studies and expression analysis have identified transforming growth factor- $\beta$ (TGF- $\beta$ ) (BMP4 and nodal) (Conlon et al. 1994; Hogan 1996; Schier and Shen 2000) and Wnt (Yamaguchi 2001) signaling pathways as key regulators of this process. Moreover, they have shown that the gradient of these pathways established in the embryo likely has a critical role in the spatiotemporal allocation of cells to different fates. For instance, bone morphogenetic protein 4 (BMP4) expressed from the extraembryonic ectoderm is found at the highest levels in the proximal or posterior part of the embryo and at the lowest levels in a distal region that contains the anterior PS. Inhibitors of BMP signaling, including noggin and chordin, present in the anterior region contribute to the formation of this gradient (McMahon et al. 1998; Bachiller et al. 2000). Nodal forms a reverse gradient to that of BMP4. The highest concentrations of nodal are found in the node near the distal tip of the PS, whereas the lowest are detected in the posterior region of the embryo. During gastrulation, Wnt expression is restricted to the posterior region of the PS as well as to the posterior and lateral proximal epiblast. Collectively, these gradients establish signaling domains that in part regulate the establishment of specific fates. Recreating these precise signaling domains is an important consideration for generating comparable cell types from PSCs in culture (Gadue et al. 2005).

\section{RECREATING KEY ASPECTS OF GASTRULATION IN PSC DIFFERENTIATION CULTURES}

\section{Strategies for PSC Differentiation}

To achieve reproducible and efficient differentiation from PSCs, it is necessary to develop approaches to study the early stages of lineage commitment in a quantitative fashion. Reporter mESC lines in which enhanced green fluorescent protein (EGFP) cDNA has been targeted to either the $t$ or mixll locus (Fehling et al. 2003; Ng et al. 2005) have been generated to enable the investigator to monitor PS formation in differentiation cultures and to isolate cells that represent these early developmental stages. A similar MIXL1-EGFP cell line has also been generated for hESCs (Davis et al. 2008). To segregate the in-vitro-generated PS into posterior and anterior populations, a truncated human CD4 (hCD4) cDNA was targeted to the anterior PS gene foxa 2 in the $\mathrm{mESC}$ line carrying the $t$-EGFP knockin ( $t$-EGFP/foxa2- $h C D 4$ ESCs) (Gadue et al. 2006). Following PS formation, mesoderm induction can be monitored by expression of flk-1 (Ema et al. 2006), and the combination of Cxcr4 and c-Kit can be used successfully to identify early endoderm (Gouon-Evans et al. 2006).

Although early studies demonstrated the in vitro potential of ESCs using fetal bovine serum (FBS) as a differentiation inducer, these conditions were not optimal for induction of a broad range of lineages. In addition, consistency and reproducibility of differentiation often relied on specific batches of selected serum. During the past 5 years,

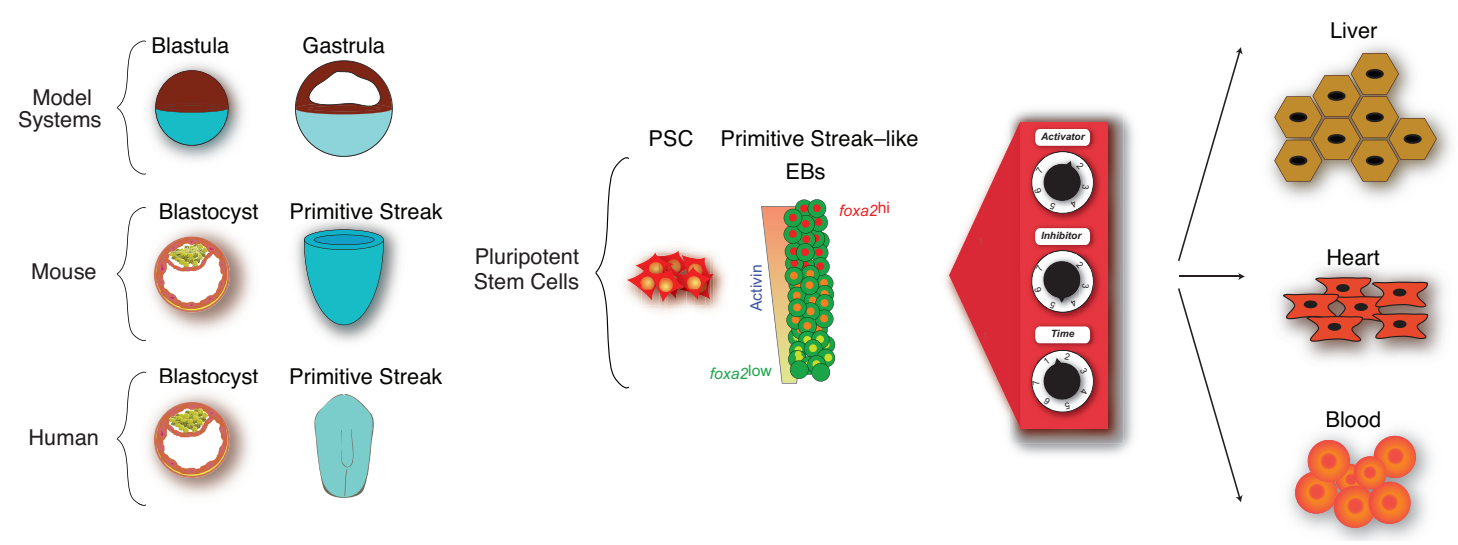

Figure 2. Translating developmental biology to pluripotent stem cell differentiation. By translating principles of development to the directed differentiation of PSCs, it is now possible to generate highly enriched populations of different cell types including immature hepatocytes, cardiomyocytes, and hematopoietic cells. The dial on the right of the figure highlights the importance of applying appropriate concentrations of agonists (activators) and antagonists (inhibitors) of key signaling pathways at the correct stage (time) of development. 
FBS has been successfully replaced with specific agonists of signaling pathways, known to function in early development. With appropriate combinations, concentrations, and timing of addition of both agonists and antagonists of these pathways, it is possible to recapitulate many of the early events associated with gastrulation in a culture dish (Fig. 2).

\section{PS Formation from PSCs}

Using the dual reporter $t-E G F P /$ foxa2-hCD4 ESC line, it has been possible to investigate the signaling requirements for generation of a PS-like population in culture. By monitoring EGFP and hCD4 expression, we demonstrated that both Wnt and nodal signaling are simultaneously required for the development of the population that coexpresses $t$ and foxa 2 (Gadue et al. 2006). The effects of Wnt3A and activin $\mathrm{A} /$ nodal are synergistic and result in the generation of a PSlike population composed of T-EGFP ${ }^{\text {pos }} /$ Foxa2-hCD4 ${ }^{\text {high }}$, T-EGFP ${ }^{\text {pos }} /$ Foxa2-hCD4 ${ }^{\text {medium }}$, and T-EGFP ${ }^{\text {os }} /$ Foxa2$\mathrm{hCD} 4{ }^{\text {low }}$ subpopulations that display characteristics of the anterior, mid, and posterior streak, respectively (Monaghan et al. 1993). The anterior T-EGFP ${ }^{\text {pos }} /$ Foxa2-hCD $4{ }^{\text {high }}$ population gives rise to definitive endoderm and derivative cell types, whereas the mid and posterior T-EGFP ${ }^{\text {pos}} /$ Foxa2hCD4 ${ }^{\text {medium }}$ and T-EGFP ${ }^{\text {pos }} /$ Foxa2-hCD4 ${ }^{\text {low }}$ populations generate different subsets of mesoderm.

The relative size of these subpopulations can be influenced by levels of nodal signaling, with high activin-A concentrations preferentially inducing the T-EGFP ${ }^{\text {pos/ }}$ Foxa2-hCD4 ${ }^{\text {high }}$ subpopulation and low concentrations promoting the formation of the T-EGFP pos/Foxa2hCD4 ${ }^{\text {medium/low }}$ subpopulations. Although Wnt and nodal are required for PS formation, BMP signaling appears to be dispensable at this stage because blocking the pathway with soluble BMP receptors had no effect on the development of the Wnt/activin A-induced T-EGFP ${ }^{\text {pos}} /$ Foxa2hCD4 ${ }^{\text {pos }}$ population (Nostro et al. 2008). Interestingly, when added alone, BMP4 does induce a T-EGFP ${ }^{\text {pos/ }}$ Foxa2-hCD4 ${ }^{\text {low }}$ PS population; however, the effect appears to be indirect and likely mediated through the induction of the Wnt and nodal pathways as development of the population was blocked when Wnt and nodal signaling was inhibited and both Wnt3A and nodal messages were detected in the embryoid body cells (EBCs) following BMP4 induction. When added together with Wnt3A and activin A, BMP4 appears to have a dominant "posteriorizing" effect, resulting in the generation of a $\mathrm{T}$ EGFP $^{\text {pos }} /$ Foxa2-hCD $4{ }^{\text {low }}$ PS population.

Studies with hESCs suggest that the early development of a human PS is regulated in a similar fashion. BMP4 alone appears to induce the equivalent of a posterior PS population able to generate hematopoietic cells, whereas high doses of activin A promote the formation of anterior PS cells that give rise to definitive endoderm and derivative cell types (D'Amour et al. 2005; Kennedy et al. 2007; Basma et al. 2008; Davis et al. 2008). Detailed analysis revealed that activin $\mathrm{A} /$ nodal and canonical Wnt function in a synergistic fashion to induce the anterior PS population (Sumi et al. 2008), similar to the activity observed with the mESCs. Collectively, these observations with mouse and hESCs highlight two important aspects of ESC differentiation. First, they clearly demonstrate that cells from both species respond to the same signaling pathways that regulate gastrulation and PS formation in the early mouse embryo, strongly supporting the notion that PSC differentiation in culture recapitulates the key events in normal embryonic development. Second, they highlight the absolute requirement for selecting appropriate combinations and concentrations of cytokines during the initial stages of differentiation (Fig. 2).

\section{Endoderm Formation from PSCs}

Endoderm forms from epiblast cells that move through the anterior PS, in a region adjacent to the node that expresses high levels of nodal. As anterior PS cells migrate out of the streak, they give rise to a sheet of endodermal cells that subsequently forms the gut tube. Cells within the gut tube grow into a columnar epithelium that is specified by the surrounding tissue into the different organs including the thymus, thyroid, lung stomach, liver, pancreas, and intestines (Wells and Melton 1999). Studies using different animal model systems have highlighted the critical role of nodal signaling for both PS formation and endoderm development (Zhou et al. 1993; Conlon et al. 1994; Jones et al. 1995; Feldman et al. 1998; Osada and Wright 1999; Gritsman et al. 2000; Lowe et al. 2001).

A major hurdle in studying definitive endoderm differentiation in vitro has been the paucity of markers that distinguish it from visceral endoderm. Given that visceral endoderm is not derived from $\mathrm{T}^{+}$cells, we used the $t$ $E G F P / f o x a 2-h C D 4 \mathrm{ESC}$ line to follow the progression of the T-EGFP ${ }^{\text {pos }} /$ Foxa2-hCD $4{ }^{\text {high }}$ anterior PS population to a definitive endoderm population characterized by a loss of $\mathrm{T}$ expression and the retention of high levels of Foxa2hCD4. This developmental step, from anterior PS to definitive endoderm, is dependent on sustained activin A/nodal signaling, clearly demonstrating that the signaling pathways that regulate the induction of this germ layer in the early embryo also function at this stage in mESC differentiation cultures.

Formation of endoderm-derived tissues and organs in vivo is dependent on complex cellular interactions that need to be recapitulated in culture. During embryogenesis, the newly formed endoderm folds anteriorly and posteriorly to generate three distinct areas: the foregut, midgut, and hindgut. BMP4 and FGF signals, provided by the septum transversum and cardiac mesoderm, respectively, are required to pattern the foregut endoderm to a liver phenotype (Zaret 2008). Both factors have been shown to function at the level of hepatic specification of ESC-derived endoderm, indicating that the requirement of these pathways is recapitulated in the in vitro model (Gouon-Evans et al. 2006).

In addition to cardiac mesoderm and the septum transversum, the endothelial lineage also has a pivotal role in the establishment of endoderm-derived tissues. In the absence of Flk- $1^{+}$cells, the liver and pancreatic buds do not expand and thus are unable to form mature organs, suggesting that endothelial cells may have a pivotal role in proliferation and differentiation of early hepatic and pancreatic progenitors (Matsumoto et al. 2001; Yoshitomi and Zaret 
2004). Observations from ESC differentiation studies suggest that a similar interaction between endothelial cells and endoderm-derived populations may be required in vitro. When induced to differentiate to a hepatic fate, the TEGFP $^{\text {pos }} /$ Foxa2-hCD $4^{\text {high }} / \mathrm{c}-\mathrm{Kit}^{\text {high }}$ PS cells generate a population consisting of large distinct clusters or colonies of $\alpha$-fetoprotein(afp) ${ }^{\text {pos }}$, albumin(alb) ${ }^{\text {pos }}$, and Foxa2-hCD4 $4^{\text {pos }}$ hepatic-like cells surrounded by $\mathrm{CD} 31^{+}$endothelial cells (Gouon-Evans et al. 2006). Fluorescence-activated cell sorting (FACS) analysis revealed that the endothelial and endodermal cells represent approximately $40 \%$ and $50 \%$ of the population, respectively (Fig. 3, top). The close spatial development of the endothelial cells and maturing hepatocytes suggests some interaction between the two lineages. Evidence in support of this was provided by additional studies which showed that anterior PS cells isolated from the activin-A-induced EBCs on the basis of c-Kit and Cxcr4 gave rise to the endodermal population (Foxa2$\mathrm{hCD} 4^{\text {pos }}$ ) but did not generate the endothelial cells $\left(\mathrm{CD} 31^{\text {pos }}\right.$ ) (Fig. 3, bottom). Maturation along the hepatic lineage did proceed in the absence of the endothelial cells, but the total cell yield in these cultures was significantly less than in the cultures containing both lineages generated from the T-EGFP ${ }^{\text {pos }} /$ Foxa2-hCD 4 high/c-Kit ${ }^{\text {high }}$ population (Gouon- Evans et al. 2006). These findings clearly document a role for endothelial cells in the development of the ESC-derived hepatic lineage in ESC cultures. Access to these populations at the earliest stages of induction in the ESC model provides an unprecedented opportunity to further investigate the interactions between the endothelial cells and the maturing hepatic lineage.

The developmental biology approach has also been successfully applied to the differentiation of hESCs to endoderm and derivative cell types. Using a step-wise differentiation approach aimed at recreating specific developmental stages within the pancreatic lineage, D'Amour et al. (2006) were able to generate insulin-producing $\beta$

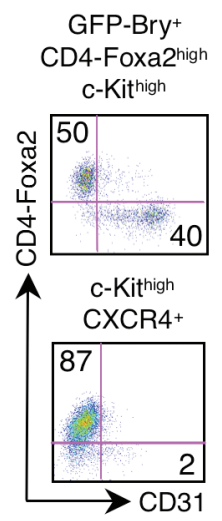

Figure 3. Endothelial potential of GFP-Bry ${ }^{+} / \mathrm{CD} 4-\mathrm{Foxa} 22^{\text {high }} / \mathrm{c}-$ $\mathrm{Kit}^{\text {high }}$ (T-GFP ${ }^{\text {pos }} /$ Foxa2-hCD4 ${ }^{\text {pos }} / \mathrm{c}^{-\mathrm{Ki}^{\mathrm{pos}}}$ ) and c-Kit ${ }^{\text {high }} / \mathrm{CXCR} 4+$ $\left(\mathrm{Cxcr}^{\mathrm{pos}} / \mathrm{c}-\mathrm{Kit}^{\mathrm{pos}}\right)$ populations. T-GFP ${ }^{\text {pos }} /$ Foxa2-hCD4 $4^{\text {pos }} / \mathrm{c}-\mathrm{Kit}^{\text {pos }}$ (top) and $\mathrm{Cxcr} 4^{\text {pos }} / \mathrm{c}-\mathrm{Kit}^{\text {pos }}$ (bottom) were isolated by cell sorting from 4-day-old EBCs induced with high concentrations of activin A. The sorted cells were plated on gelatin and analyzed 3 days later for the presence of endothelial CD $31^{+}$and endodermal (Foxa2$\mathrm{hCD} 4^{\mathrm{pos}}$ ) cells. (Reprinted, with permission, from Gouon-Evans et al. 2006 [@ Nature Publishing Group].) cells from hESCs. Whereas the cells formed in the cultures did not display all of the characteristics of functional $\beta$ cells, the study further highlights the importance of translating findings from developmental biology to the in vitro differentiation of PSCs.

\section{Mesoderm}

Understanding the specific stages of mesoderm development and the signaling pathways that regulate them is a prerequisite for generating appropriate derivative cell types from PSCs in vitro. Subpopulations of mesoderm fated to form different tissues are generated by distinct subsets of epiblast cells that egress through the posterior, mid, and anterior regions of the primitive streak. Access to markers that distinguish these early populations will be important for isolating specific mesoderm-derived lineages from PSCs. Although extensive marker sets do not yet exist to measure mesoderm induction and specification, the receptor tyrosine kinase Flk-1 (vascular endothelial growth factor receptor-2, VEGFR-2) has been used to monitor some of the early differentiation stages. Flk-1 was originally identified as an endothelial-cell-specific receptor (Millauer et al. 1993; Quinn et al. 1993; Yamaguchi et al. 1993). However, expression analyses of early embryo and lineage-tracing studies uncovered a broader pattern before endothelial specification and demonstrated expression in mesoderm fated to the hematopoietic, vascular, cardiac, and somitic lineages (Millauer et al. 1993; Quinn et al. 1993; Yamaguchi et al. 1993; Motoike et al. 2003; Ema et al. 2006).

To formally demonstrate that Flk-1 is a valid marker for mesoderm induction, we evaluated the potential of the population to give rise to nonmesoderm cell types, specifically endoderm following exposure to high concentrations of activin A. Posterior PS cells that have not yet up-regulated Flk-1 (Flk-1 ${ }^{\text {neg }} /$ T-EGFP $^{\text {pos }} /$ Foxa2-hCD4 ${ }^{\text {neg }}$ ) will differentiate to Foxa2-hCD4 ${ }^{\text {pos }}$ endoderm when cultured in activin A for 24 hours, indicating that their fate is not yet fixed to the mesoderm lineage. In contrast, the Flk-1 ${ }^{\text {pos }} /$ T-EGFP ${ }^{\text {pos }} /$ Foxa2-hCD4 ${ }^{\text {neg }}$ population isolated from the same stage EBCs appears to have lost the potential to generate endoderm, because it does not give rise to Foxa2-hCD4 ${ }^{\text {pos }}$ cells following culture with activin A. Rather, this population up-regulated expression of the hematopoietic-vascular marker CD31 under these conditions, strongly suggesting that its mesodermal fate is fixed and that Flk-1 is a valid marker for this developmental step (Nostro et al. 2008).

\section{Hematopoietic Mesoderm}

The blood islands of the yolk sac represent the onset of hematopoiesis in the mammalian embryo and derive from the first mesodermal cell population to egress from the PS (Russell 1979). These structures are composed of clusters of primitive erythroblasts surrounded by developing endothelial cells and are present in the presumptive yolk sac by E7.5 of mouse development (Haar and Ackerman 1971). The close temporal and spatial development of the hematopoietic and endothelial lineages in blood islands provided the basis for the hypothesis that these lineages 
develop from a common ancestor, a cell known as the hemangioblast (Sabin 1917; Murray 1932). Although gene expression and targeting studies in different model systems provided evidence that supported the concept of the hemangioblast (Keller 2005), it was studies with the mESC model that demonstrated the existence of this progenitor (Choi et al. 1998; Nishikawa et al. 1998). The mESC-derived hemangioblasts, also known as blast colony-forming cells (BL-CFCs), express Flk-1 and T, develop early in differentiation cultures, and are able to generate blast cell colonies that contain hematopoietic, endothelial, and vascular smooth-muscle potential (Choi et al. 1998; Fehling et al. 2003). The emergence of this progenitor within the differentiation cultures, before the establishment of all other blood cell lineages, indicates that it defines the earliest stage of hematopoietic commitment. On the basis of findings from the ESC studies, we were able to identify a comparable progenitor in the early embryo (Huber et al. 2004). This cell also expresses Flk1 and T, develops in the posterior PS of the E7.0 embryo, and is able to generate progeny of the hematopoietic, endothelial, and vascular smooth-muscle lineages. The potential of this progenitor strongly suggests that it represents the in vivo hemangioblast, the progenitor of the yolk sac hematopoietic system. A progenitor with hemangioblast characteristics has also been identified in hESC differentiation cultures (Kennedy et al. 2007). The human hemangioblast also expresses KDR (Flk-1), displays the potential to generate both hematopoietic and endothelial progeny, and emerges within the cultures before the establishment of other hematopoietic lineages. Collectively, these studies highlight two important aspects of PSC differentiation. First, they demonstrate the power of the in vitro system for studying early developmental stages that are difficult to access in the mammalian embryo. Second, they provide strong evidence that lineage development in the ESC model follows that of the early embryo and that information gained from the mESC model can be translated to the mouse embryo as well as to the human system.

The identification of the Flk-1 ${ }^{\text {pos }} / \mathrm{T}_{-\mathrm{EGFP}}{ }^{\mathrm{pos}}$ hemangioblast as the earliest stage of hematopoietic commitment in the ESC system was important because it provided a readily detectable marker for quantifying blood cell commitment. By monitoring T-EGFP, Flk-1, and the hemato- poietic-specific marker CD41 (Ferkowicz et al. 2003; Mikkola et al. 2003), we were able to map the stage-specific signaling requirements in the developmental pathway from ESCs to blood (Nostro et al. 2008). The outcome of this study showed that a combination of Wnt and activin/nodal signaling were required for the initial step, the formation of the T-EGFP pos PS population. Wnt, activin/ nodal, and BMP4 were essential for induction of Flk-1 ${ }^{\text {pos }}$ hematopoietic mesoderm from the PS, whereas VEGF/Flk1 signaling was required to specify this population to a hematopoietic fate. Further detailed analysis revealed that Wnt, produced endogenously by the Flk-1 ${ }^{\text {pos }}$ mesoderm, is essential for specification of the primitive erythroid lineage. This effect of Wnt appeared to be specific to primitive erythropoieis because the macrophage lineage, which also develops at this early stage, was not affected (Fig. 4).

More recent studies using the ESC model have shown that primitive erythroid specification is more complex and is regulated by cross-talk between the Wnt and Notch signaling pathways. Notch signaling inhibits primitive erythroid specification in vivo as well as in ESC differentiation cultures in vitro and therefore must be inhibited during primitive erythroid development (Fig. 4) (Hadland et al. 2004; Cheng et al. 2008). Numb, a Notch inhibitor, present in the early yolk sac as well as in developing hemangioblast colonies in culture likely functions in this capacity. The demonstration that the combination of enforced expression of numb together with activation of Wnt signaling displayed a synergistic effect on primitive erythroid development supports this interpretation (Cheng et al. 2008). Collectively, these findings provide the first detailed insights into the regulation of the primitive erythroid lineage, the first blood lineage to develop, and demonstrate that Wnt signaling has a pivotal role at this developmental step.

\section{Cardiac Mesoderm}

Cardiac mesoderm is derived from epiblast cells that move through the PS at a more distal site and at a slightly later time point than the hemangioblast mesoderm fated to colonize the yolk sac (Parameswaran and Tam 1995). Following induction, the cardiac mesodermal population migrates to the anterior region of the embryo where it is

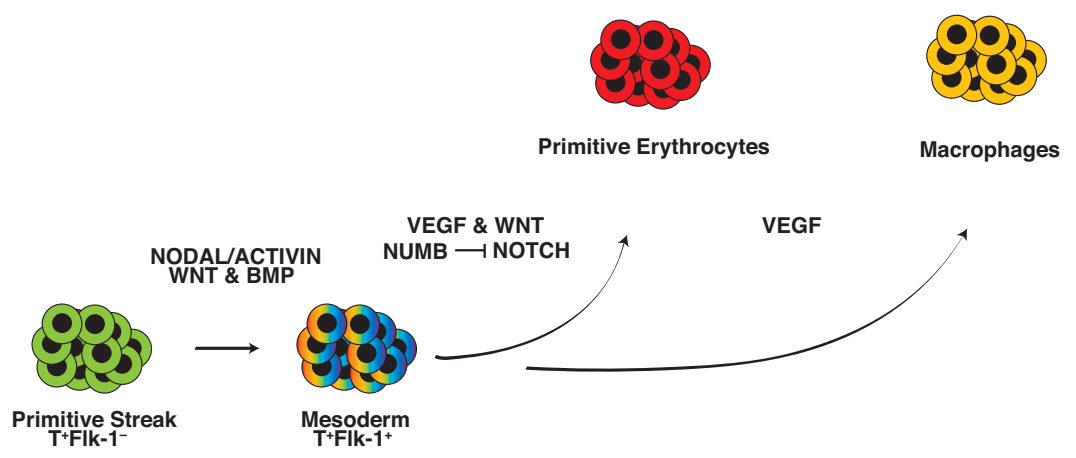

Figure 4. Model of primitive hematopoiesis during ESC differentiation. BMP4, nodal/activin A, and Wnt signaling are all necessary for the induction of a mesoderm population $\left(\mathrm{T}^{+} \mathrm{Flk}-1^{+}\right)$from primitive streak-like cells $\left(\mathrm{T}^{+} \mathrm{Flk}-\right.$. Wnt, VEGF, and numb function in concert to induce the primitive erythroid lineage from the Flk- $1^{+}$mesoderm. VEGF alone is sufficient to induce the macrophage lineage from the same population. 
exposed to cytokines of the BMP (Schultheiss et al. 1997) and FGF (Reifers et al. 2000) families as well as inhibitors of the Wnt pathway (Marvin et al. 2001; Schneider and Mercola 2001; Tzahor and Lassar 2001). This signaling environment specifies the mesodermal cells to a cardiac fate, leading to the formation of the cardiac crescent that gives rise to the primitive heart tube consisting of cardiac (myocardium) and endothelial (endocardium) cells.

To study the earliest stages of cardiac development in the ESC differentiation system, we developed a strategy to recreate in vitro the temporal allocation of mesoderm fates observed in the early embryo (Kouskoff et al. 2005). Using the $t$-EGFP reporter cell line, we identified the emergence of two distinct Flk-1 ${ }^{\text {pos }} /$ T-EGFP ${ }^{\text {pos }}$ populations at different times in the ESC cultures (Kattman et al. 2006). The first Flk-1 ${ }^{\text {pos}} /$ T-EGFP ${ }^{\text {pos }}$ population to develop contained hemangioblasts, whereas the second population was enriched for cardiac potential. Detailed analyses of the second cardiogenic Flk-1 ${ }^{\text {pos }} /$ T-EGFP ${ }^{\text {pos }}$ population revealed that it contained cardiovascular progenitors that displayed endothelial, cardiomyocyte, and vascular smooth-muscle development. A comparable progenitor population was identified in the early mouse embryo, suggesting that the cardiovascular lineages in vivo and in vitro derive from a Flk- $1^{\text {pos }}$ progenitor. The concept of a common Flk- $1^{\text {pos }}$ cardiovascular progenitor is supported by the studies of Moretti et al. (2006), who described a Flk- ${ }^{\text {pos }} / \mathrm{Isl1}^{\text {pos }}$ population with similar potential, and by gene targeting and lineage-tracing studies showing that cells of the cardiac lineage express the receptor or are derived from cells that had expressed it (Motoike et al. 2003; Yamashita et al. 2005; Ema et al. 2006). The cardiovascular progenitors develop before the establishment of cardiac lineage, indicating that they define the onset of cardiovascular development in this model.

The identification of the cardiovascular progenitor in mESC cultures provided the basis for the identification of a comparable human progenitor (Yang et al. 2008). By recapitulating the signaling environment of the cardiac region of the mouse embryo in hESC differentiation cultures, we were able to establish conditions that promoted the efficient and reproducible development of the cardiac lineage from the hESCs. Analysis of the EBCs at different stages led to the identification of a distinct $\mathrm{KDR}^{\text {pos}}$ (Flk-1) population between day five and six of differentiation that displayed cardiovascular potential. The cardiogenic $\mathrm{KDR}^{\text {pos }}$ population was easily distinguished from the hematopoietic/vascular $\mathrm{KDR}^{\text {pos }}$ population by lack of c-KIT expression. As observed in the mouse system, the human cardiogenic $\mathrm{KDR}^{\mathrm{pos}}$ population contained cardiovascular progenitors that displayed cardiac, endothelial, and vascular smooth-muscle potential.

The identification of cardiovascular progenitors in both mouse and human cultures highlights important similarities between these species in the establishment of lineages that participate in the formation of the first organs to develop in the early embryo. Access to these progenitors provides an enriched source of cardiomyocytes for in vitro and in vivo studies as well as a unique opportunity for investigating the molecular pathways that regulate the specification of the cardiac, endothelial, and vascular smooth-muscle lineages that comprise three of the major cell types of the adult heart.

\section{Genetic Modification of PSCs}

The ability to genetically alter hPSCs is a prerequisite for creating reporter cell lines to monitor specific developmental steps, for establishing gain- and loss-of-function approaches to investigate the molecular mechanisms regulating early human development, and ultimately for repairing genetic mutations in patient-specific iPSCs. With mESCs, HR has been the most widely used method for genetic modification because it enables the investigator to either create or repair mutations as well as to generate reporter ESC lines and mice with confidence that the transgene will faithfully recapitulate expression of the endogenous locus. In contrast to the routine use of this technology in mESCs, its application to hESCs has been limited (Zwaka and Thomson 2003; Irion et al. 2007; Davis et al. 2008). Although detailed protocols of the entire strategy have been published addressing some of the technical differences between mouse and human ESCs, the methodology has not translated well to the human system (Zwaka and Thomson 2003; Costa et al. 2007).

The identification of specific loci that allow broad expression of targeted genetic material (reporter genes, cDNAs) has had an important role in a wide variety of developmental biology and genetic studies in the mouse. The most notable of these loci is Rosa26, identified originally through a gene-trap approach (Zambrowicz et al. 1997). Rosa26 is a preferred site for genetic modification because it is expressed in most tissues throughout development and in adult life and is relatively easy to target through homologous recombination. Using a bioinformatics approach, we identified a locus in the human genome that shared marked homology with the mouse Rosa26 sequence (Irion et al. 2007). The locus is broadly expressed in fetal and adult tissues and can be targeted through HR using a gene-trap approach, comparable to the one routinely used to target the mouse locus (Soriano 1999; Luche et al. 2007). Importantly, the tandem-dimer red fluorescent protein (tdRFP) (Campbell et al. 2002) reporter introduced into human ROSA26 was expressed in the undifferentiated $\mathrm{hESCs}$ as well as in derivatives of all three germ layers generated during the in vitro differentiation of these cells. There was no evidence of silencing of the locus following long periods of culture of the undifferentiated cells or following their differentiation to derivative lineages (Fig. 5). The targeting vector contained two loxP sites (one wild type and one mutant) that created a "docking" site in the ROSA26 locus for introduction of new genetic material through recombinase-mediated cassette exchange (RMCE) (Bouhassira et al. 1997). Using RMCE, it was possible to replace the tdRFP cDNA with other sequences including the EGFP and puromycin resistance cDNAs. Strategies to introduce vectors that allow the inducible expression of transgenes from the ROSA26 locus, now under way, will provide a model system for using gain- and loss-of-genefunction approaches to study human development.

Given that the standard approaches for HR can be slow and have met with limited success in the human system, other state-of-the-art technologies for genetic modification of hPSCs must be considered. One interesting approach is the use of zinc finger nucleases (ZFNs) that can dramati- 

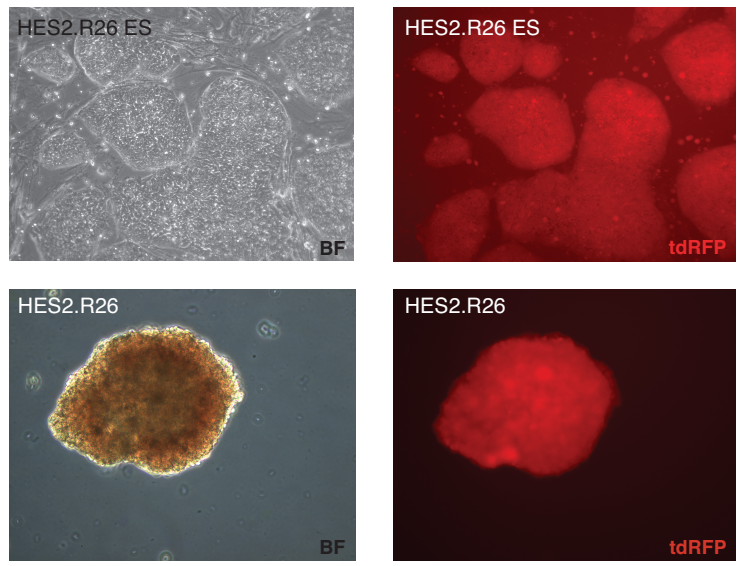

cally increase the efficiency of HR through directing double-strand breaks to the desired target gene (Urnov et al. 2005; Lombardo et al. 2007). When ZFNs are delivered to the cells together with a conventional targeting construct, $\mathrm{HR}$ is significantly more efficient compared to a random double-strand break, possibly alleviating the need for drug selection (Santiago et al. 2008). Other approaches that may improve the efficiency of HR in hESCs include the use of adeno-associated viruses to target a specific sequence in the human genome. Proof-of-principle studies in the mouse have demonstrated that this strategy can be used to correct a small deletion in the Rosa 26 locus (Miller et al. 2004, 2006).

In addition to $\mathrm{HR}$, other approaches that rely on random integration of the introduced genetic material in hPSCs are being developed and optimized. These include the use of lentiviral vectors (Lombardo et al. 2007), bacterial artificial chromosomes (Placantonakis et al. 2008), the sleeping beauty transposon system (Wilber et al. 2007), and the $\phi$ C31 integrase (Thyagarajan et al. 2008). All have been successfully used to genetically modify mammalian cells and all have their own unique advantages and disadvantages. As the field matures and as these and other technologies yet to be developed are evaluated, the efficient and genetic modification of hPSCs will be become a routine practice in most labs.

\section{ACKNOWLEDGMENTS}

We thank members of the Keller lab for many helpful discussions and a critical reading of the manuscript. This work was supported by grants from the National Institutes of Health (G.M.K., S.J.K.) and the Leopoldina Stiftung der Naturforscher (S.I.).

\section{REFERENCES}

Bachiller, D., Klingensmith, J., Kemp, C., Belo, J.A., Anderson, R.M., May, S.R., McMahon, J.A., McMahon, A.P., Harland, R.M., Rossant, J., et al. 2000. The organizer factors Chordin and Noggin are required for mouse forebrain development. Nature 403: 658-661.

Basma, H., Soto-Gutierrez, A., Yannam, G.R., Liu, L., Ito, R., Yamamoto, T., Ellis, E., Carson, S.D., Sato, S., Chen, Y., et al. 2008. Differentiation and transplantation of human embry-
Figure 5. Genetic modification of human embryonic stem cells. HES2 hESCs were targeted at the ROSA26 locus with a targeting construct harboring a red fluorescent protein (tdRFP) cDNA. Under bright-field microscopy (BF, upper left) these cells show normal morphology and display robust expression of tdRFP when examined by fluorescent microscopy (tdRFP, upper right). The targeted cells are able to differentiate into the hematopoietic lineage, as demonstrated by the development of an erythroid colony in methylcellulose cultures (BF, lower left). The cells within the erythroid colony express tdRFP (tdRFP, lower right), demonstrating that expression of the reporter is maintained following differentiation of the targeted hESCs. onic stem cell-derived hepatocytes. Gastroenterology 3: 990-999.

Bouhassira, E.E., Westerman, K., and Leboulch, P. 1997. Transcriptional behavior of LCR enhancer elements integrated at the same chromosomal locus by recombinase-mediated cassette exchange. Blood 90: 3332-3344.

Campbell, R.E., Tour, O., Palmer, A.E., Steinbach, P.A., Baird, G.S., Zacharias, D.A., and Tsien, R.Y. 2002. A monomeric red fluorescent protein. Proc. Natl. Acad. Sci. 99: 7877-7882.

Cheng, X., Huber, T.L., Chen, V.C., Gadue, P., and Keller, G.M. 2008. Numb mediates the interaction between Wnt and Notch to modulate primitive erythropoietic specification from the hemangioblast. Development 135: 3447-3458.

Choi, K., Kennedy, M., Kazarov, A., Papadimitriou, J.C., and Keller, G. 1998. A common precursor for hematopoietic and endothelial cells. Development 125: 725-732.

Conlon, F.L., Lyons, K.M., Takaesu, N., Barth, K.S., Kispert, A., Herrmann, B., and Robertson, E.J. 1994. A primary requirement for nodal in the formation and maintenance of the primitive streak in the mouse. Development 120: 1919-1928.

Costa, M., Dottori, M., Sourris, K., Jamshidi, P., Hatzistavrou, T., Davis, R., Azzola, L., Jackson, S., Lim, S.M., Pera, M., Elefanty, A.G., and Stanley, E.G. 2007. A method for genetic modification of human embryonic stem cells using electroporation. Nat. Protoc. 2: 792-796.

D’Amour, K.A., Agulnick, A.D., Eliazer, S., Kelly, O.G., Kroon, E., and Baetge, E.E. 2005. Efficient differentiation of human embryonic stem cells to definitive endoderm. Nat. Biotechnol. 23: 1534-1541.

D'Amour, K.A., Bang, A.G., Eliazer, S., Kelly, O.G., Agulnick, A.D., Smart, N.G., Moorman, M.A., Kroon, E., Carpenter, M.K., and Baetge, E.E. 2006. Production of pancreatic hormone-expressing endocrine cells from human embryonic stem cells. Nat. Biotechnol. 24: 1392-1401.

Davis, R.P., Ng, E.S., Costa, M., Mossman, A.K., Sourris, K., Elefanty, A.G., and Stanley, E.G. 2008. Targeting a GFP reporter gene to the MIXL1 locus of human embryonic stem cells identifies human primitive streak-like cells and enables isolation of primitive hematopoietic precursors. Blood 111: $1876-1884$.

Dush, M.K. and Martin, G.R. 1992. Analysis of mouse Evx genes: $E v x-1$ displays graded expression in the primitive streak. Dev. Biol. 151: 273-287.

Ema, M., Takahashi, S., and Rossant, J. 2006. Deletion of the selection cassette, but not cis-acting elements, in targeted Flk1-lacZ allele reveals Flk1 expression in multipotent mesodermal progenitors. Blood 107: 111-117.

Evans, M. and Kaufman, M. 1981. Establishment in culture of pluripotent cells from mouse embryos. Nature 292: 154-156.

Fehling, H.J., Lacaud, G., Kubo, A., Kennedy, M., Robertson, S., Keller, G., and Kouskoff, V. 2003. Tracking mesoderm induction and its specification to the hemangioblast during embry- 
onic stem cell differentiation. Development 130: 4217-4227.

Feldman, B., Gates, M.A., Egan, E.S., Dougan, S.T., Rennebeck, G., Sirotkin, H.I., Schier, A.F., and Talbot, W.S. 1998. Zebrafish organizer development and germ-layer formation require nodal-related signals. Nature 395: 181-185.

Ferkowicz, M.J., Starr, M., Xie, X., Li, W., Johnson, S.A., Shelley, W.C., Morrison, P.R., and Yoder, M.C. 2003. CD41 expression defines the onset of primitive and definitive hematopoiesis in the murine embryo. Development 130: 4393-4403.

Forlani, S., Lawson, K.A., and Deschamps, J. 2003. Acquisition of Hox codes during gastrulation and axial elongation in the mouse embryo. Development 130: 3807-3819.

Gadue, P., Huber, T.L., Paddison, P.J., and Keller, G.M. 2006 Wnt and TGF- $\beta$ signaling are required for the induction of an in vitro model of primitive streak formation using embryonic stem cells. Proc. Natl. Acad. Sci. 103: 16806-16811.

Gadue, P., Huber, T.L., Nostro, M.C., Kattman, S., and Keller, G.M. 2005. Germ layer induction from embryonic stem cells. Exp. Hematol. 33: 955-964.

Glaser, S., Anastassiadis, K., and Stewart, A.F. 2005. Current issues in mouse genome engineering. Nat. Genet. 37: 11871193.

Gouon-Evans, V., Boussemart, L., Gadue, P., Nierhoff, D., Koehler, C.I., Kubo, A., Shafritz, D.A., and Keller, G. 2006. BMP-4 is required for hepatic specification of mouse embryonic stem cell-derived definitive endoderm. Nat. Biotechnol. 24: $1402-1411$.

Gritsman, K., Talbot, W.S., and Schier, A.F. 2000. Nodal signaling patterns the organizer. Development 127: 921-932.

Haar, J.L. and Ackerman, G.A. 1971. A phase and electron microscopic study of vasculogenesis and erythropoiesis in the yolk sac of the mouse. Anat. Rec. 170: 199-223.

Hadland, B.K., Huppert, S.S., Kanungo, J., Xue, Y., Jiang, R., Gridley, T., Conlon, R.A., Cheng, A.M., Kopan, R., and Longmore, G.D. 2004. A requirement for Notch1 distinguishes 2 phases of definitive hematopoiesis during development. Blood 104: 3097-3105.

Hart, A.H., Hartley, L., Sourris, K., Stadler, E.S., Li, R., Stanley, E.G., Tam, P.P., Elefanty, A.G., and Robb, L. 2002. Mixll is required for axial mesendoderm morphogenesis and patterning in the murine embryo. Development 129: 3597-3608.

Hogan, B.L. 1996. Bone morphogenetic proteins in development. Curr. Opin. Genet. Dev. 6: 432-438.

Huber, T.L., Kouskoff, V., Fehling, H.J., Palis, J., and Keller, G. 2004. Haemangioblast commitment is initiated in the primitive streak of the mouse embryo. Nature 432: 625-630.

Irion, S., Luche, H., Gadue, P., Fehling, H.J., Kennedy, M., and Keller, G.M. 2007. Identification and targeting of the ROSA26 locus in human embryonic stem cells. Nat. Biotechnol. 25: $1477-1482$

Jones, C.M., Kuehn, M.R., Hogan, B.L., Smith, J.C., and Wright, C.V. 1995. Nodal-related signals induce axial mesoderm and dorsalize mesoderm during gastrulation. Development 121: 3651-3662.

Kattman, S.J., Huber, T.L., and Keller, G.M. 2006. Multipotent flk- $1^{+}$cardiovascular progenitor cells give rise to the cardiomyocyte, endothelial, and vascular smooth muscle lineages. Dev. Cell 11: 723-732.

Keller, G. 2005. Embryonic stem cell differentiation: Emergence of a new era in biology and medicine. Genes Dev. 19: 11291155.

Kennedy, M., D’Souza, S.L., Lynch-Kattman, M., Schwantz, S., and Keller, G. 2007. Development of the hemangioblast defines the onset of hematopoiesis in human ES cell differentiation cultures. Blood 109: 2679-2687.

Kinder, S.J., Tsang, T.E., Quinlan, G.A., Hadjantonakis, A.K., Nagy, A., and Tam, P.P. 1999. The orderly allocation of mesodermal cells to the extraembryonic structures and the anteroposterior axis during gastrulation of the mouse embryo. Development 126: 4691-4701.

Kinder, S.J., Tsang, T.E., Wakamiya, M., Sasaki, H., Behringer, R.R., Nagy, A., and Tam, P.P. 2001. The organizer of the mouse gastrula is composed of a dynamic population of progenitor cells for the axial mesoderm. Development 128: 3623
3634.

Kispert, A. and Herrmann, B.G. 1994. Immunohistochemical analysis of the Brachyury protein in wild-type and mutant mouse embryos. Dev. Biol. 161: 179-193.

Kouskoff, V., Lacaud, G., Schwantz, S., Fehling, H.J., and Keller, G. 2005. Sequential development of hematopoietic and cardiac mesoderm during embryonic stem cell differentiation. Proc. Natl. Acad. Sci. 102: 13170-13175.

Li, W., Wei, W., Zhu, S., Zhu, J., Shi, Y., Lin, T., Hao, E., Hayek, A., Deng, H., and Ding, S. 2009. Generation of rat and human induced pluripotent stem cells by combining genetic reprogramming and chemical inhibitors. Cell Stem Cell 4: 16-19.

Lombardo, A., Genovese, P., Beausejour, C.M., Colleoni, S., Lee, Y.L., Kim, K.A., Ando, D., Urnov, F.D., Galli, C., Gregory, P.D., Holmes, M.C., and Naldini, L. 2007. Gene editing in human stem cells using zinc finger nucleases and integrasedefective lentiviral vector delivery. Nat. Biotechnol. 25: 12981306.

Lowe, L.A., Yamada, S., and Kuehn, M.R. 2001. Genetic dissection of nodal function in patterning the mouse embryo. Development 128: 1831-1843.

Luche, H., Weber, O., Nageswara Rao, T., Blum, C., and Fehling, H.J. 2007. Faithful activation of an extra-bright red fluorescent protein in "knock-in" Cre-reporter mice ideally suited for lineage tracing studies. Eur. J. Immunol. 37: 43-53.

Martin, G.R. 1981. Isolation of a pluripotent cell line from early mouse embryos cultured in medium conditioned by teratocarcinoma stem cells. Proc. Natl. Acad. Sci. 78: 7634-7638.

Marvin, M.J., Di Rocco, G., Gardiner, A., Bush, S.M., and Lassar, A.B. 2001. Inhibition of Wnt activity induces heart formation from posterior mesoderm. Genes Dev. 15: 316-327.

Matsumoto, K., Yoshitomi, H., Rossant, J., and Zaret, K.S. 2001. Liver organogenesis promoted by endothelial cells prior to vascular function. Science 294: 559-563.

McMahon, J.A., Takada, S., Zimmerman, L.B., Fan, C.M., Harland, R.M., and McMahon, A.P. 1998. Noggin-mediated antagonism of BMP signaling is required for growth and patterning of the neural tube and somite. Genes Dev. 12: 1438-1452.

Mikkola, H.K., Fujiwara, Y., Schlaeger, T.M., Traver, D., and Orkin, S.H. 2003. Expression of CD41 marks the initiation of definitive hematopoiesis in the mouse embryo. Blood 101: 508-516.

Millauer, B., Wizigmann-Voos, S., Schnurch, H., Martinez, R., Moller, N.P., Risau, W., and Ullrich, A. 1993. High affinity VEGF binding and developmental expression suggest Flk-1 as a major regulator of vasculogenesis and angiogenesis. Cell 72: $835-846$.

Miller, D.G., Petek, L.M., and Russell, D.W. 2004. Adeno-associated virus vectors integrate at chromosome breakage sites. Nat. Genet. 36: 767-773.

Miller, D.G., Wang, P.R., Petek, L.M., Hirata, R.K., Sands, M.S., and Russell, D.W. 2006. Gene targeting in vivo by adeno-associated virus vectors. Nat. Biotechnol. 24: 1022-1026.

Monaghan, A.P., Kaestner, K.H., Grau, E., and Schutz, G. 1993. Postimplantation expression patterns indicate a role for the mouse forkhead/HNF-3 $\alpha, \beta$ and $\gamma$ genes in determination of the definitive endoderm, chordamesoderm and neuroectoderm. Development 119: 567-578.

Moretti, A., Caron, L., Nakano, A., Lam, J.T., Bernshausen, A., Chen, Y., Qyang, Y., Bu, L., Sasaki, M., Martin-Puig, S., et al. 2006. Multipotent embryonic $i s l 1^{+}$progenitor cells lead to cardiac, smooth muscle, and endothelial cell diversification. Cell 127: 1151-1165.

Motoike, T., Markham, D.W., Rossant, J., and Sato, T.N. 2003. Evidence for novel fate of $\mathrm{Flk1}^{+}$progenitor: Contribution to muscle lineage. Genesis 35: 153-159.

Murray, P.D.F. 1932. The development of in vitro of the blood of the early chick embryo. Proc. Roy. Soc. Lond. 11: 497-521.

Ng, E.S., Azzola, L., Sourris, K., Robb, L., Stanley, E.G., and Elefanty, A.G. 2005. The primitive streak gene Mixll is required for efficient haematopoiesis and BMP4-induced ventral mesoderm patterning in differentiating ES cells. Development 132: 873-884.

Nishikawa, S.I., Nishikawa, S., Hirashima, M., Matsuyoshi, N., 
and Kodama, H. 1998. Progressive lineage analysis by cell sorting and culture identifies FLK1 ${ }^{+}$VE-cadherin ${ }^{+}$cells at a diverging point of endothelial hematopoietic lineages. Development 125: $1747-1757$.

Nostro, M.C., Cheng, X., Keller, G.M., and Gadue, P. 2008. Wnt, activin, and BMP signaling regulate distinct stages in the developmental pathway from embryonic stem cells to blood. Cell Stem Cell 2: 60-71.

Osada, S.I. and Wright, C.V. 1999. Xenopus nodal-related signaling is essential for mesendodermal patterning during early embryogenesis. Development 126: 3229-3240.

Parameswaran, M. and Tam, P.P. 1995. Regionalisation of cell fate and morphogenetic movement of the mesoderm during mouse gastrulation. Dev. Genet. 17: 16-28.

Park, I.H., Zhao, R., West, J.A., Yabuuchi, A., Huo, H., Ince, T.A., Lerou, P.H., Lensch, M.W., and Daley, G.Q. 2008. Reprogramming of human somatic cells to pluripotency with defined factors. Nature 451: 141-146.

Placantonakis, D.G., Tomishima, M.J., Lafaille, F., Desbordes, S.C., Jia, F., Socci, N.D., Viale, A., Lee, H., Harrison, N., Tabar, V., et al. 2008. BAC transgenesis in human ES cells as a novel tool to define the human neural lineage. Stem Cells (in press).

Quinn, T., Peters, K., De Vries, C., Ferrara, N., and Williams, L. 1993. Fetal liver kinase 1 is a receptor for vascular endothelial growth factor and is selectively expressed in vascular endothelium. Proc. Natl. Acad. Sci. 90: 7533-7537.

Reifers, F., Walsh, E.C., Leger, S., Stainier, D.Y., and Brand, M. 2000. Induction and differentiation of the zebrafish heart requires fibroblast growth factor 8 (fgf 8 /acerebellar). Development 127: 225-235.

Russell, E. 1979. Heriditary anemias of the mouse: A review for geneticists. Adv. Genet. 20: 357-459.

Sabin, F.R. 1917. Origin and development of the primitive vessels of the chick and of the pig. Contrib. Embryol. Carnegie Inst. 226: 61-124.

Santiago, Y., Chan, E., Liu, P.Q., Orlando, S., Zhang, L., Urnov, F.D., Holmes, M.C., Guschin, D., Waite, A., Miller, J.C., et al. 2008. Targeted gene knockout in mammalian cells by using engineered zinc-finger nucleases. Proc. Natl. Acad. Sci. 105: $5809-5814$

Sasaki, H. and Hogan, B.L. 1993. Differential expression of multiple forkhead related genes during gastrulation and axial pattern formation in the mouse embryo. Development 118: 47-59.

Schier, A.F. and Shen, M.M. 2000. Nodal signalling in vertebrate development. Nature 403: 385-389.

Schneider, V.A. and Mercola, M. 2001. Wnt antagonism initiates cardiogenesis in Xenopus laevis. Genes Dev. 15: 304-315.

Schultheiss, T.M., Burch, J.B., and Lassar, A.B. 1997. A role for bone morphogenetic proteins in the induction of cardiac myogenesis. Genes Dev. 11: 451-462.

Soriano, P. 1999. Generalized lacZ expression with the ROSA26 Cre reporter strain. Nat. Genet. 21: 70-71.

Stevens, L.C. and Little, C.C. 1954. Spontaneous testicular teratomas in an inbred strain of mice. Proc. Natl. Acad. Sci. 40: $1080-1087$.

Sumi, T., Tsuneyoshi, N., Nakatsuji, N., and Suemori, H. 2008. Defining early lineage specification of human embryonic stem cells by the orchestrated balance of canonical Wnt/ $\beta$ catenin, activin/nodal and BMP signaling. Development 135: 2969-2979.

Takahashi, K. and Yamanaka, S. 2006. Induction of pluripotent stem cells from mouse embryonic and adult fibroblast cultures by defined factors. Cell 126: 663-676.

Takahashi, K., Tanabe, K., Ohnuki, M., Narita, M., Ichisaka, T.,
Tomoda, K., and Yamanaka, S. 2007. Induction of pluripotent stem cells from adult human fibroblasts by defined factors. Cell 131: 861-872.

Tam, P.P. and Behringer, R.R. 1997. Mouse gastrulation: The formation of a mammalian body plan. Mech. Dev. 68: 3-25.

Thomson, J.A., Itskovitz-Eldor, J., Shapiro, S.S., Waknitz, M.A., Swiergiel, J.J., Marshall, V.S., and Jones, J.M. 1998. Embryonic stem cell lines derived from human blastocysts. Science 282: 1145-1147.

Thyagarajan, B., Liu, Y., Shin, S., Lakshmipathy, U., Scheyhing, K., Xue, H., Ellerstrom, C., Strehl, R., Hyllner, J., Rao, M.S., and Chesnut, J.D. 2008. Creation of engineered human embryonic stem cell lines using phiC31 integrase. Stem Cells 26: 119-126.

Tzahor, E. and Lassar, A.B. 2001. Wnt signals from the neural tube block ectopic cardiogenesis. Genes Dev. 15: 255-260.

Urnov, F.D., Miller, J.C., Lee, Y.L., Beausejour, C.M., Rock, J.M., Augustus, S., Jamieson, A.C., Porteus, M.H., Gregory, P.D., and Holmes, M.C. 2005. Highly efficient endogenous human gene correction using designed zinc-finger nucleases. Nature 435: 646-651.

Wells, J.M. and Melton, D.A. 1999. Vertebrate endoderm development. Annu. Rev. Cell Dev. Biol. 15: 393-410.

Wilber, A., Linehan, J.L., Tian, X., Woll, P.S., Morris, J.K., Belur, L.R., McIvor, R.S., and Kaufman, D.S. 2007. Efficient and stable transgene expression in human embryonic stem cells using transposon-mediated gene transfer. Stem Cells 25: 2919-2927.

Yamaguchi, T.P. 2001. Heads or tails: Wnts and anterior-posterior patterning. Curr. Biol. 11: R713-R724.

Yamaguchi, T.P., Dumont, D.J., Conlon, R.A., Breitman, M.L., and Rossant, J. 1993. flk-1, an flt-related receptor tyrosine kinase is an early marker for endothelial cell precursors. Development 118: 489-498.

Yamashita, J.K., Takano, M., Hiraoka-Kanie, M., Shimazu, C., Peishi, Y., Yanagi, K., Nakano, A., Inoue, E., Kita, F., and Nishikawa, S. 2005. Prospective identification of cardiac progenitors by a novel single cell-based cardiomyocyte induction. FASEB J. 19: 1534-1536.

Yang, L., Soonpaa, M.H., Adler, E.D., Roepke, T.K., Kattman, S.J., Kennedy, M., Henckaerts, E., Bonham, K., Abbott, G.W., Linden, R.M., Field, L.J., and Keller, G.M. 2008. Human cardiovascular progenitor cells develop from a $\mathrm{KDR}^{+}$embryonicstem-cell-derived population. Nature 453: 524-528.

Yoshitomi, H. and Zaret, K.S. 2004. Endothelial cell interactions initiate dorsal pancreas development by selectively inducing the transcription factor Ptfla. Development 131: 807-817.

Yu, J., Vodyanik, M.A., Smuga-Otto, K., Antosiewicz-Bourget, J., Frane, J.L., Tian, S., Nie, J., Jonsdottir, G.A., Ruotti, V., Stewart, R., et al. 2007. Induced pluripotent stem cell lines derived from human somatic cells. Science 318: 1917-1920.

Zambrowicz, B.P., Imamoto, A., Fiering, S., Herzenberg, L.A., Kerr, W.G., and Soriano, P. 1997. Disruption of overlapping transcripts in the ROSA $\beta$ geo 26 gene trap strain leads to widespread expression of $\beta$-galactosidase in mouse embryos and hematopoietic cells. Proc. Natl. Acad. Sci. 94: 3789-3794.

Zaret, K.S. 2008. Genetic programming of liver and pancreas progenitors: Lessons for stem-cell differentiation. Nat. Rev. Genet. 9: 329-340.

Zhou, X., Sasaki, H., Lowe, L., Hogan, B.L., and Kuehn, M.R. 1993. Nodal is a novel TGF- $\beta$-like gene expressed in the mouse node during gastrulation. Nature 361: 543-547.

Zwaka, T.P. and Thomson, J.A. 2003. Homologous recombination in human embryonic stem cells. Nat. Biotechnol. 21: 319-321. 


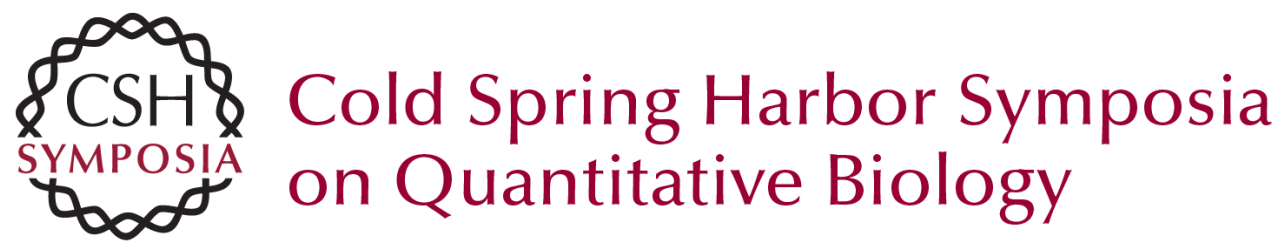

\section{Directed Differentiation of Pluripotent Stem Cells: From Developmental Biology to Therapeutic Applications}

S. Irion, M.C. Nostro, S.J. Kattman, et al.

Cold Spring Harb Symp Quant Biol 2008 73: 101-110 originally published online March 27, 2009 Access the most recent version at doi:10.1101/sqb.2008.73.065

References This article cites 90 articles, 44 of which can be accessed free at: http://symposium.cshlp.org/content/73/101.full.html\#ref-list-1

\section{License}

Email Alerting Service top right corner of the article or click here. 